\title{
Relative Convergence of the Solution of a Doubly Infinite Set of Equations
}

\author{
R. Mittra \\ Contribution from University of Illinois, Urbana, Ill., and Electromagnetics Staff, Radar \\ Division, Ground Systems Group, Hughes Aircraft Company, Fullerton, Calif.
}

(Received June 21, 1962; revised October 31, 1962)

The paper deals with the relative convergence of a doubly-infinite set of equations pertaining to a boundary value problem in a waveguide. It is shown that the solution for the equations converges differently for different choices of the combination of the number of equations from the two sets. This is demonstrated by studying the convergence properties of the truncated set of equations as the number of equations is made indefinitely large. It is proven that the solution for the reflection coefficient becomes identical with the exact solution only with a particular choice of the ratio of the equations. This choice of the unique ratio is also shown to be consistent with the edge condition.

\section{Introduction}

In formulating electromagnetic boundary value problems we are often led to an infinite set of equations. In most cases, it is not possible to invert the infinite matrix and we are forced to resort to truncating the above to a finite size. We then solve the equations for a number of increasing sizes of the matrix and study the convergence of the solution. If at least the leading members of the unknown coefficients (which are usually of primary interest) tend to converge, we feel satisfied and assume that we have obtained a reasonably good approximation for the leading coefficients.

It is the purpose of this paper to show in the first instance that for a particular doubly infinite set of equations associated with the bifurcation problem in a waveguide, there is a relative convergence of the solution, meaning that the solution converges to a different set of answers for every different choice of the ratio $C=P / Q . \quad P$ and $Q$ are the numbers of equations from the first and second set, respectively, out of the doubly infinite set of equations. These equations for the bifurcation problem have been obtained by Hurd and Gruenberg [1954], who have also presented an exact solution of the infinite set through the use of calculus of residues. The above set, because it has a known solution, is particularly suited for our purpose which is to demonstrate by considering several finite size matrices that there results a relative convergence when $P \rightarrow \infty$ and $Q \rightarrow \infty$. Furthermore, we are also able to find the ratio of $P / Q$ which yields the correct answer in the limit.

In the second part of this paper we present a basis for choosing the correct ratio of $P / Q$ when working with a truncated set. With this choice, when the size of the set is increased indefinitely while keeping the ratio $C$ constant, the solution does converge to the correct answer. It is shown through the study of the asymptotic behavior of the higher order unknown coefficients and the application of the edge condition, why only an unique choice of the ratio would make the solution asymptotically tend to the correct one and why otherwise an incorrect solution will result.

\section{Development of the Infinite Set of Equations for H-Plane Bifurcation}

The infinite set of equations which will be discussed here in connection with the problem of $H$-plane bifurdation in a rectangular waveguide has been derived by Hurd [1954]. We shall therefore skip the details and merely outline the procedure for their derivation. 

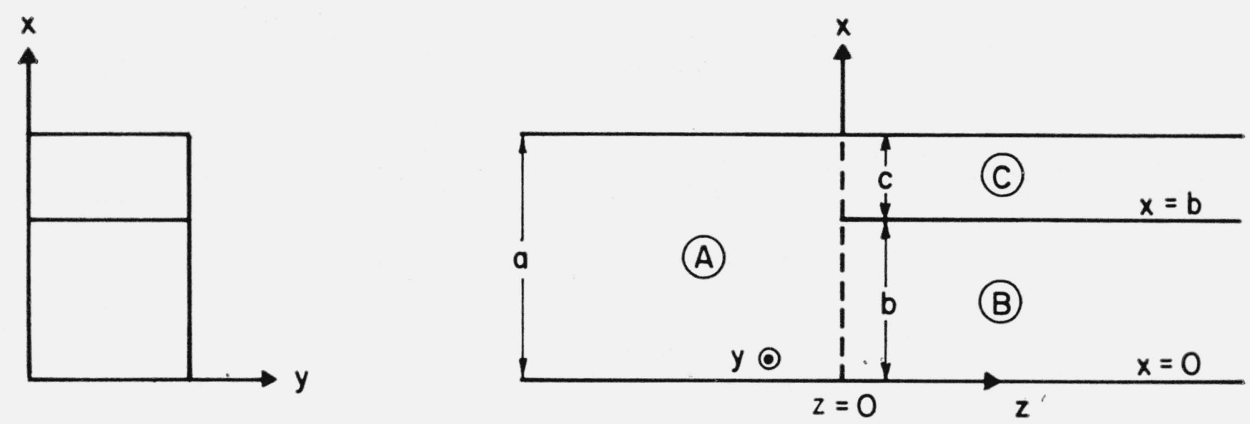

FIgURE 1. H-plane bifurcation of a rectangular waveguide.

The geometry under consideration is shown in figure 1. Assume that the incident wave from the negative $z$-direction is a $\mathrm{TE}_{10}$ mode with the electric vector parallel to the edge of the septum. It can be easily shown that the only nonzero field components are $E_{y}, H_{x}$, and $H_{z}$ and that they can all be expressed in terms of a scalar function $\varphi=E_{y}$ and its partial derivatives.

The problem can be stated in terms of the equation

$$
\nabla_{x z}^{2} \varphi+k^{2} \varphi=0
$$

and the following boundary conditions on $\varphi$ :

(a) $\varphi$ and $\nabla \varphi$ are finite everywhere in the region concerned, except at the edge of the bifurcation at $z=0, x=b$ where $\nabla \varphi$ becomes infinite.

(b) $\varphi$ and $\nabla \varphi$ are continuous in the subregions and at $z=0$.

(c) $\varphi$ behaves as an outgoing wave at large $z$ apart from the incident field.

(d) $\varphi$ vanishes on the walls $x=0, a$ and on the septum at $x=b, z>0$.

(e) $\varphi$ satisfies the edge condition and hence goes to zero at the edge as $r^{1 / 2}$, where $r$ is the distance from the edge. $\nabla \varphi$ therefore goes to infinity as $r^{-1 / 2}$ as $r \rightarrow 0$.

It is easily verified that $\varphi_{A}, \varphi_{B}$ and $\varphi_{C}$ expressed in the following equations satisfy (1) and the conditions $(a),(c)$, and $(d)$. The expressions are:

$$
\begin{aligned}
& \varphi_{A}=A e^{-\alpha_{1}^{z}} \sin (\pi x / a)+\sum_{n=1}^{\infty} A_{n} e^{\alpha} n^{z} \sin (n \pi x / a) \\
& \varphi_{B}=\sum_{n=1}^{\infty} B_{n} e^{-\beta_{n}^{2}} \sin (n \pi x / b) \\
& \varphi_{C}=\sum_{n=1}^{\infty} C_{n} e^{-\gamma} n^{z} \sin n \pi(x-b) / c
\end{aligned}
$$

where

$$
\begin{aligned}
A & =\text { amplitude of the incident field } \\
\alpha_{n} & =\left[(n \pi / a)^{2}-k^{2}\right]^{1 / 2} \\
\beta_{n} & =\left[(n \pi / b)^{2}-k^{2}\right]^{1 / 2} \\
\gamma_{n} & =\left[(n \pi / c)^{2}-k^{2}\right]^{1 / 2} \\
k & =2 \pi / \lambda, \lambda=\text { free space wavelength. }
\end{aligned}
$$

$\alpha, \beta$, and $\gamma$ 's are the mode propagation constants in the three regions.

By applying the continuity conditions at $z=0$ and subsequently equating the Fourier components of the resulting equations in the range $0<x<b$ and $b<x<c$, one arrives at the following doubly infinite set of equations after some manipulations (for details see Hurd [1954]): 


$$
\begin{array}{ll}
\sum_{n=1}^{\infty} \frac{A_{n} \sin (n \pi b / a)}{\alpha_{n}-\beta_{p}}=\frac{A \sin (\pi b / a)}{\alpha_{1}+\beta_{p}} & p=1, \ldots \infty \\
\sum_{n=1}^{\infty} \frac{A_{n} \sin (n \pi b / a)}{\alpha_{n}-\gamma_{q}}=\frac{A \sin (\pi b / a)}{\alpha_{1}+\gamma_{q}} & q=1, \ldots \infty
\end{array}
$$

where $\delta_{n}^{1}=0$ for $n \neq 1$ and equals 1 when $n=1$.

The set of infinite equations (3) has been solved exactly by Hurd. We shall, however, concentrate on the solution of the truncated set of equations for various combinations of $P$ and $Q$ where these are the number of equations from the set (3a) and (3b) respectively. We proceed to do this in the following section.

\section{Solution of Truncated Set of Equations}

Consider the solution of the truncated set of equations

$$
\begin{array}{ll}
\sum_{n=1}^{P+Q} \frac{A_{n} \sin (n \pi b / a)}{\alpha_{n}-\beta_{p}}=\frac{A \sin (\pi b / a)}{\alpha_{1}+\beta_{p}} & p=1,2, \ldots P \\
\sum_{n=1}^{P+Q} \frac{A_{n} \sin (n \pi b / a)}{\alpha_{n}-\gamma_{q}}=\frac{A \sin (\pi b / a)}{\alpha_{1}+\gamma_{q}} & q=1,2, \ldots Q
\end{array}
$$

In attempting to solve the set of equations (4) we recognize first of all that the determinant of the equation is of a particular kind which is called a double alternant [Muir, 1960]. Written explicitly, the determinant is

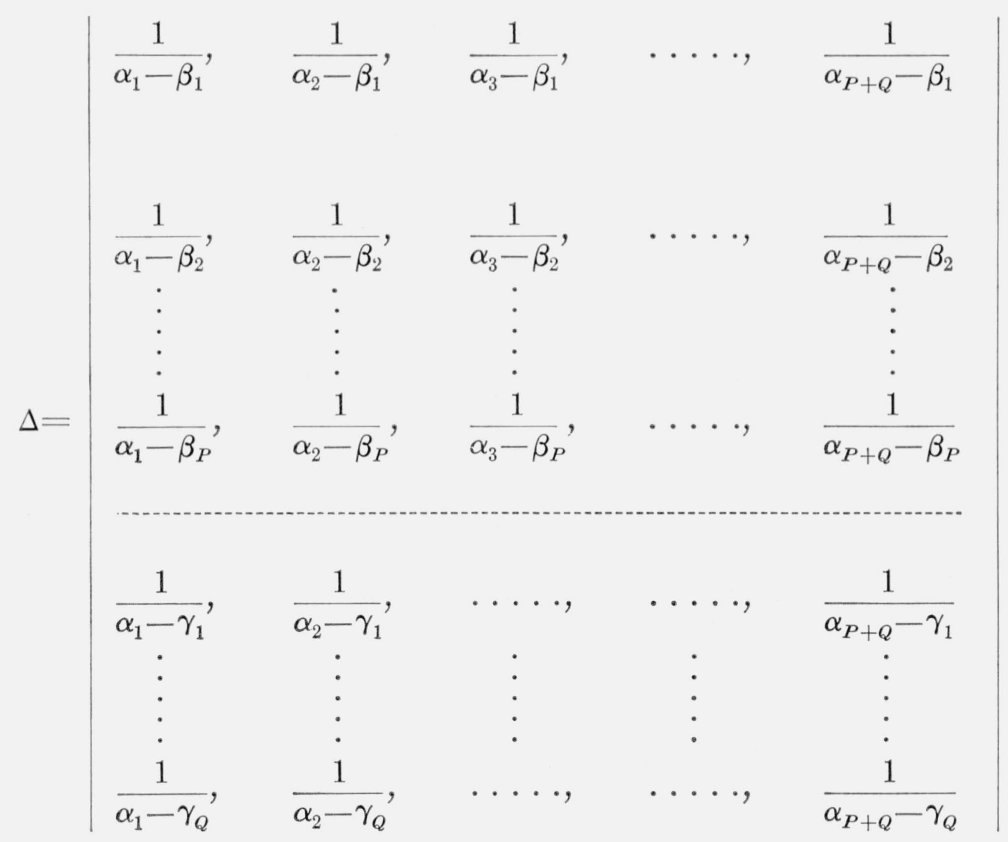

Note that we have grouped the factors $\sin (n \pi b / a)$ with the unknowns $A_{n}$.

It is observed that each element of the determinant is a reciprocal of the difference of two quantities, $\alpha_{r}$ and $\beta_{s}$ or $\gamma_{t}$ in general, only one of which, viz, the $\alpha$, changes as one goes along the columns, whereas only $\beta$ or $\gamma$ changes as one goes down the rows. Hence, if $\alpha_{r} \rightarrow \alpha_{n}$, where $r$ and $n$ are two different subscripts, then it is obvious that the determinant $\Delta \rightarrow 0$ and that the zero is a simple one. This is effective to saying that $\left(\alpha_{r}-\alpha_{n}\right)$ for various combinations of $r$ and $n$ are the factors in the continued product expansion of the determinant. In a similar manner, we observe that $\left(\beta_{r}-\beta_{n}\right),\left(\gamma_{r}-\gamma_{n}\right)$ and $\left(\beta_{t}-\gamma_{s}\right)$ are also to be included in the product 
expansion of the determinant. We have to make sure, however, that all such factors are included in the product and that none is repeated. It is also obvious that the denominator of this expression for $\Delta$ must contain the factors $\left(\alpha_{t}-\beta_{s}\right)$ and $\left(\alpha_{t}-\gamma_{r}\right)$ for various combinations of the subscripts. The expression for the $\Delta$ developed on the basis of above arguments, is

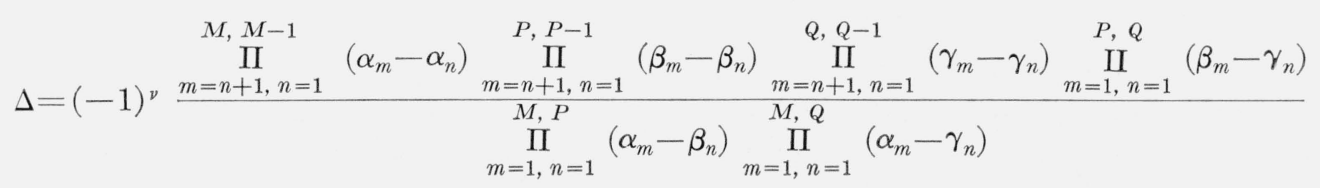

where $M=P+Q ; \nu$ is an even or odd integer, depending on $P$ and $Q$ and as yet undetermined, although we shall not really need to find $\nu$. This is because in order to calculate the coefficients $A_{r}$ we need find only the ratio of the determinants $\Delta_{r} / \Delta$ where $\Delta_{r}$ is the determinant obtained by replacing the $r$ th column of $\Delta$ by the column representing the right-hand side of (4). It is fairly straightforward that

$$
A_{r} \sin (r \pi b / a)=-\frac{A}{\Delta} \Delta\left(\alpha_{r} \rightarrow-\alpha_{1}\right) \sin (\pi b / a)
$$

where $\Delta\left(\alpha_{r} \rightarrow-\alpha_{1}\right)$ is the determinant $\Delta$ with $\alpha_{r}$ replaced by $-\alpha_{1}$. We can therefore obtain through the use of (6) and (7) an expression for $A_{\tau}$ in the product form, which is

$$
\begin{aligned}
A_{r} \frac{\sin (r \pi b / a)}{\sin (\pi b / a)} & =-A \frac{\Delta\left(\alpha_{r} \rightarrow-\alpha_{1}\right)}{\Delta} \\
& =-A \prod_{n=1}^{P} \frac{\left(\beta_{n}-\alpha_{r}\right)}{\left(\beta_{n}+\alpha_{1}\right)} \prod_{n=1}^{Q} \frac{\left(\gamma_{n}-\alpha_{r}\right)}{\left(\gamma_{n}+\alpha_{1}\right)} \cdot \prod_{n=1}^{M(r)} \frac{\left(\alpha_{n}+\alpha_{1}\right)}{\left(\alpha_{n}-\alpha_{r}\right)}
\end{aligned}
$$

where the superscript $(r)$ on the last product implies that the factor corresponding to $n=r$ is to be omitted. Equation (8) gives the desired solution for the truncated matrix. In particular, the expression for $A_{1} / A$, which can be identified as the reflection coefficient $R$, is

$$
\begin{aligned}
R=\frac{A_{1}}{A} & =-\prod_{n=1}^{P} \frac{\left(\beta_{n}-\alpha_{1}\right)}{\left(\alpha_{1}+\beta_{n}\right)} \prod_{n=1}^{Q} \frac{\left(\gamma_{n}-\alpha_{1}\right)}{\left(\alpha_{1}+\gamma_{n}\right)} \prod_{n=2}^{M} \frac{\left(\alpha_{n}+\alpha_{1}\right)}{\left(\alpha_{n}-\alpha_{1}\right)} \\
& =-\prod_{n=1}^{P} \frac{\left(1-\alpha_{1} / \beta_{n}\right)}{\left(1+\alpha_{1} / \beta_{n}\right)} \prod_{n=1}^{Q} \frac{\left(1-\alpha_{1} / \gamma_{n}\right)}{\left(1+\alpha_{1} / \gamma_{n}\right)} \prod_{n=2}^{M} \frac{\left(1+\alpha_{1} / \alpha_{n}\right)}{\left(1-\alpha_{1} / \alpha_{n}\right)} .
\end{aligned}
$$

We also quote below for comparison and reference, the result arrived at by Hurd as a solution of the infinite set of equations. His expression has a sign error and corrected it reads

$R=-\frac{\Pi^{(1)}\left(-\alpha_{1}, \alpha\right) \Pi\left(\alpha_{1}, \beta\right) \Pi\left(\alpha_{1}, \gamma\right)}{\Pi^{(1)}\left(\alpha_{1}, \alpha\right) \Pi\left(-\alpha_{1}, \beta\right) \Pi\left(-\alpha_{1}, \gamma\right)} \times \exp \left[\left(2 \alpha_{1} / \pi\right)\{-a+b \ln (a / b)+c \ln (a / c)\}\right]$

where for instance

$$
\Pi(\omega, \alpha)=\prod_{p=1}^{\infty}\left(\alpha_{p}-\omega\right)(a / p \pi) e^{(a \omega / p \pi)}
$$

and the superscript (1) implies as before that the factor corresponding to $p=1$ is to be omitted.

\section{Comparison of Solutions of Finite and Infinite Set of Equations}

In this section, we shall study the asymptotic behavior of the expression for the reflection coefficient $R$. We shall see that even when $(P+Q)$ is very large, the expression $(9)$ for the reflection coefficient yields different values for different ratios of $P / Q$. We shall then compare the answer for a particular choice of $P / Q$ and see that it indeed converges to the exact value of $R$ calculated through the use of (10). 
Consider the particular case when all the mode propagation constants excepting $\alpha_{1}$ are real and $\alpha_{1}$ is purely imaginary. This is done merely for convenience, and the conclusions regarding the convergence phenomenon reached in this case will still be applicable to the general case when the above condition is not true.

For this case, it is obvious from (9) that $R=e^{j \theta}$, i.e., $|R|=1$. The angle $\theta=$ argument $R$ is given by

$$
\theta=\arg R=\pi-2\left[\sum_{n=1}^{P} \tan ^{-1}\left(\delta / \beta_{n}\right)+\sum_{n=1}^{Q} \tan ^{-1}\left(\delta / \gamma_{n}\right)-\sum_{n=2}^{P+Q} \tan ^{-1}\left(\delta / \alpha_{n}\right)\right]
$$

where $\alpha_{1}=j \delta$. But since $\beta_{n}=\sqrt{(n \pi / b)^{2}-k^{2}}$ and $\delta=\sqrt{k^{2}-(\pi / a)^{2}}$, one can write

$$
\tan ^{-1} \delta / \beta_{n}=\sin ^{-1}\left\{(b \delta / \pi) /\left(n^{2}-b^{2} / a^{2}\right)^{1 / 2}\right\} .
$$

After similarly expressing the other arctans in terms of arc sines, one can rewrite (11) as

$$
\frac{\theta}{2}=\frac{\pi}{2}+\sum_{n=2}^{P+Q} \sin ^{-1} \frac{(a \delta / \pi)}{\sqrt{n^{2}-1}}-\sum_{n=1}^{P} \sin ^{-1} \frac{(b \delta / \pi)}{\sqrt{n^{2}-(b / a)^{2}}}-\sum_{n=1}^{Q} \sin ^{-1} \frac{(c \delta / \pi)}{\sqrt{n^{2}-(c / a)^{2}}} .
$$

A similar expression for the exact $\theta_{e x}$ has been derived from (10) and is given in the following:

$$
\frac{\theta_{e x}}{2}=\frac{\pi}{2}-\frac{a \delta}{\pi}\left(1-\frac{b}{a} \ln \frac{a}{b}-\frac{c}{a} \ln \frac{a}{c}\right)+S_{2}\left(\frac{\delta a}{\pi} ; 1,0\right)-S_{1}\left(\frac{\delta b}{\pi} ; \frac{b}{a}, 0\right)-S_{1}\left(\frac{\delta c}{\pi} ; \frac{c}{a}, 0\right)
$$

where

$$
S_{N}(u ; v, 0)=\sum_{n=N}^{\infty}\left[\sin ^{-1} \frac{u}{\left(n^{2}-v^{2}\right)^{1 / 2}}-\frac{u}{n}\right]
$$

We shall now go on to show that the expression for the reflection coefficient derived from the truncated set of equations becomes identical with the exact solution as $P \rightarrow \infty, Q \rightarrow \infty$, provided we choose $P / Q=b / c$. To this end, consider (12) which gives the angle of the reflection coefficient $\theta$ in terms of the arc sine series. Rewrite the expression for $\theta$ in (12) as

$$
\begin{aligned}
\frac{\theta}{2}=\frac{\pi}{2}+\left\{\sum_{n=2}^{P+Q} \sin ^{-1}\right. & \left.\left(\frac{a \delta / \pi}{\sqrt{n^{2}-1}}\right)-\frac{a \delta}{\pi} \sum_{n=2}^{P+Q} \frac{1}{n}\right\}-\left\{\sum_{n=1}^{P} \sin ^{-1}\left(\frac{b \delta / \pi}{\sqrt{n^{2}-b^{2} / a^{2}}}\right)-\frac{b \delta}{\pi} \sum_{n=1}^{P} \frac{1}{n}\right\} \\
& -\left\{\sum_{n=1}^{Q} \sin ^{-1} \frac{c \delta / \pi}{\sqrt{n^{2}-(c / a)^{2}}}-\frac{c \delta}{\pi} \sum_{n=1}^{Q} \frac{1}{n}\right\}-\frac{a \delta}{\pi}+\frac{a \delta}{\pi} \sum_{n=1}^{P+Q} \frac{1}{n}-\frac{b \delta}{\pi} \sum_{n=1}^{P} \frac{1}{n}-\frac{c \delta}{\pi} \sum_{n=1}^{Q} \frac{1}{n} .
\end{aligned}
$$

Now, use the well known relation [Magnus and Oberhetinger, 1954],

$$
\lim _{M \rightarrow \infty}\left(\sum_{n=1}^{M} \frac{1}{n}-\ln M\right)=\gamma=\text { Euler's Constant }
$$

in (14) after letting $P \rightarrow \infty, Q \rightarrow \infty$, and derive

$$
\begin{aligned}
\lim _{\substack{P \rightarrow \infty \\
Q \rightarrow \infty}} \frac{\theta}{2}=\frac{\pi}{2}-\frac{a \delta}{\pi}+S_{2}(\delta a / \pi ; 1,0)-S_{1}(\delta b / \pi ; b / a, 0)- & S_{1}(\delta c / \pi ; c / a, 0) \\
& +\lim _{\substack{P \rightarrow \infty \\
Q \rightarrow \infty}}\left\{\frac{a \delta}{\pi} \ln (P+Q)-\frac{b \delta}{\pi} \ln P-\frac{c \delta}{\pi} \ln Q\right\} .
\end{aligned}
$$

If we now let $P / Q=b / c$, we can readily derive

$$
\lim _{\substack{P \rightarrow \infty \\ Q \rightarrow \infty, P / Q=\frac{\boldsymbol{\iota}}{c}}} \theta / 2=\frac{\pi}{2}-a \delta / \pi\left[1-\frac{b}{a} \ln \frac{a}{b}-\frac{c}{a} \ln \frac{a}{c}\right]+S_{2}(\delta a / \pi ; 1,0)-S_{1}(\delta b / \pi ; b / a, 0)-S_{1}(\delta c / \pi ; c / a, 0) .
$$


Comparison shows that the limiting value of $\theta$ agrees identically with (13), which is the exact expression given by Hurd [1954].

It is also interesting to note that if $P$ and $Q$ are increased indefinitely, such that $C=P / Q$ does not equal $b / c$, the expression for $\theta$ approaches the limit

$\lim _{\substack{P \rightarrow \infty \\ Q \rightarrow \infty}} \frac{\theta}{2}=\frac{\pi}{2}-\frac{a \delta}{\pi}\left[1-\frac{b}{a} \ln \left(1+\frac{1}{C}\right)-\frac{c}{a} \ln (1+C)\right]+S_{2}(\delta a / \pi ; 1,0)-S_{1}(\delta b / \pi ; b / a, 0)-S_{1}(\delta c / \pi ; c / a, 0)$,

which is different from the correct solution. Hence, even though the solution for the reflection coefficient will converge to a limit when larger and larger size truncated matrices are taken, the convergence is relative and the solution obtained will be incorrect except for the unique choice of the ratio $P / Q=b / c$. In what follows, we go on to demonstrate that this particular choice of $P / Q$ ratio is also consistent with the criterion based on the edge condition at the edge of the bifurcation. To this end, we first study the behavior of the higher order coefficients, $A_{r}$, in the following section.

\section{Asymptotic Behavior of Higher Order Coefficients}

In this section, starting from (8), we shall develop an asymptotic expression for $A_{r} / A$ for large $P$ and $Q$.

Let us rewrite (8) as

$$
A_{r}^{\prime}=\prod_{n=1}^{P} \frac{\left(1-\alpha_{r} / \beta_{n}\right)}{\left(1+\alpha_{1} / \beta_{n}\right)} \prod_{n=1}^{Q} \frac{\left(1-\alpha_{r} / \gamma_{n}\right)}{\left(1+\alpha_{1} / \gamma_{n}\right)} \prod_{n=1}^{P+Q(r)} \frac{\left(1+\alpha_{1} / \alpha_{n}\right)}{\left(1-\alpha_{r} / \alpha_{n}\right)}
$$

where

$$
A_{r}^{\prime}=-\left(A_{r} / A\right) \frac{\sin r \pi b / a}{\sin \pi b / a}
$$

Since

$$
\beta_{n} \rightarrow \frac{n \pi}{b}, \gamma_{n} \rightarrow \frac{n \pi}{c} \text { and } \alpha_{n} \rightarrow \frac{n \pi}{a} \text { for large } n,
$$

it is convenient to introduce some additional factors in the numerator and denominator of (18) and rearrange it as

$$
\begin{aligned}
& A_{r}^{\prime}=\left\{\prod_{n=1}^{P} \frac{\left(1-\alpha_{r} / \beta_{n}\right)}{\left(1-\alpha_{r} b / n \pi\right)} \prod_{n=1}^{Q} \frac{\left(1-\alpha_{r} / \gamma_{n}\right)}{\left(1-\alpha_{r} c / n \pi\right)} \prod_{n=1}^{P+Q(r)} \frac{\left(1-\alpha_{r} a / n \pi\right)}{\left(1-\alpha_{r} / \alpha_{n}\right)}\right\} \\
& \cdot\left\{\prod_{n=1}^{P} \frac{\left(1+\alpha_{1} b / n \pi\right)}{\left(1+\alpha_{1} / \beta_{n}\right)} \prod_{n=1}^{Q} \frac{\left(1+\alpha_{1} c / n \pi\right)}{\left(1+\alpha_{1} / \gamma_{n}\right)} \prod_{n=1}^{P+Q(r)} \frac{\left(1+\alpha_{1} / \alpha_{n}\right)}{\left(1+\alpha_{1} a / n \pi\right)}\right\} \\
& \cdot \frac{\left(1-\alpha_{r} a / r \pi\right)}{\left(1+\alpha_{1} a / r \pi\right)} \frac{\prod_{n=1}^{P}\left(1-\alpha_{r} b / n \pi\right) \prod_{n=1}^{Q}\left(1-\alpha_{r} c / n \pi\right)}{\prod_{n=1}^{P+Q}\left(1-\alpha_{r} a / n \pi\right)} \cdot \frac{\prod_{n=1}^{P+Q}\left(1+\alpha_{1} a / n \pi\right)}{\prod_{n=1}^{P}\left(1+\alpha_{1} b / n \pi\right) \prod_{n=1}^{Q}\left(1+\alpha_{1} c / n \pi\right)} \cdot
\end{aligned}
$$

It may be shown that the factors inside the first two (braces) tend to constants as $P$ and $Q$ are increased indefinitely for a given $r$. This is proven in appendix 1 .

It will therefore be sufficient to study the behavior of the ratio of the products $F$, where

$$
F=\frac{\prod_{n=1}^{P}\left(1-\alpha_{r} b / n \pi\right) \prod_{n=1}^{Q}\left(1-\alpha_{r} c / n \pi\right)}{\prod_{n=1}^{P+Q}\left(1-\alpha_{r} a / n \pi\right)}\left(1-\alpha_{r} a / r \pi\right),
$$

and of a similar ratio of products with $-\alpha_{1}$ replacing $\alpha_{\tau}$. 
Let us rewrite (20) as

$$
F=\left(1-\alpha_{r} a / r \pi\right) \frac{P^{\alpha_{r} b / \pi} \prod_{n=1}^{P}\left(1-\alpha_{r} b / n \pi\right) Q^{\alpha_{r} c / \pi} \prod_{n=1}^{Q}\left(1-\alpha_{r} c / n \pi\right)}{(P+Q)^{\alpha_{r} a / \pi} \prod_{n=1}^{P+Q}\left(1-\alpha_{r} a / n \pi\right)} \frac{(P+Q)^{\alpha} r^{u / \pi}}{P^{\alpha_{r} b / \pi} Q^{\alpha_{r} c / \pi}}
$$

and then use the following representation [Magnus and Oberhetinger, 1954] of $1 / \Gamma(z+1)$ where $\Gamma(x)$ is the Gamma function of argument $x$.

$$
\frac{1}{\Gamma(z+1)}=\lim _{m \rightarrow \infty} m^{-z} \prod_{n=1}^{m}(1+z / n),
$$

to recast (20) into its asymptotic form for large $P$ and $Q$. We derive using (22) in (21), and letting $P / Q=C$ (constant),

$$
\lim _{\substack{P \rightarrow \infty \\ Q \rightarrow \infty}} F=\left(1-\alpha_{r} a / r \pi\right) \frac{\Gamma\left(1-\alpha_{r} a / \pi\right)}{\Gamma\left(1-\alpha_{r} b / \pi\right) \Gamma\left(1-\alpha_{r} c / \pi\right)} \cdot(1+1 / C)^{\alpha_{r} b / \pi}(1+C)^{\alpha_{r} c / \pi} .
$$

Next, using [Magnus and Oberhetinger, 1954]

$$
\Gamma(1-z)=\frac{\pi}{\sin \pi z \Gamma(z)}
$$

in (23) one derives

$$
\lim _{\substack{P \rightarrow \infty \\ Q \rightarrow \infty}} F=(1 / \pi) \cdot\left(1-\alpha_{r} a / r \pi\right) \cdot \frac{\sin \left(\alpha_{r} b\right) \sin \left(\alpha_{r} c\right)}{\sin \alpha_{r} a} \cdot \frac{\Gamma\left(\alpha_{r} b / \pi\right) \Gamma\left(\alpha_{r} c / \pi\right)}{\Gamma\left(\alpha_{r} a / \pi\right)} \cdot(1+1 / C)^{\alpha_{r} b / \pi}(1+C)^{\alpha_{r} c / \pi} .
$$

The next step is to study the limit of $F$ as $r$, hence $\alpha_{r}$ becomes very large. We have from Stirling's formula,

$$
\Gamma(z) \sim(2 \pi)^{1 / 2} \frac{z^{z}}{z^{1 / 2}} e^{-z} \text { for a large } z \text {. }
$$

Using (25) in (24) and letting $\alpha_{r} \rightarrow r \pi / a$ we find

$$
F \rightarrow L K_{r} \frac{\left(\frac{r b}{a}\right)^{r b / a}\left(\frac{r c}{a}\right)^{r c / a}}{(r)^{r}} \cdot \frac{1}{r^{3 / 2}}(1+1 / C)^{r b / a}(1+C)^{r c / a} \text { for large } r, P, \text { and } Q,
$$

where $L$ is a constant independent of $r$ and $K_{r}$ is an oscillatory function of $r$ such that $K_{r}-f \rightarrow 0$ and $\left|K_{r}\right|<1$. Note that we have used the fact that $\left\{1-\left(\alpha_{r} a / \pi\right) / r\right\} / \sin \alpha_{r} a$ has the limit $(-)^{r_{1}}$ $(1 / r \pi)$ as $\alpha_{r} \rightarrow r \pi / a$.

Writing

we obtain from (26)

$$
r^{r}=\left(\frac{r b}{a}+\frac{r c}{a}\right)^{r b / a+r c / a}
$$

$$
F \rightarrow \frac{L K_{r}}{r^{3 / 2}} \frac{(1+1 / C)^{r b / a}(1+C)^{r c / a}}{(1+c / b)^{r b / a}(1+b / c)^{r c / a}}
$$

for $P / Q=C, P \rightarrow \infty, Q \rightarrow \infty$, and $r$ large.

Equation (27) gives the desired asymptotic behavior of $F$. It is not difficult to show that the ratio of products

$$
\frac{1}{\left(1+\alpha_{1} a / r \pi\right)} \frac{\prod_{n=1}^{P+Q}\left(1+\alpha_{1} a / n \pi\right)}{\prod_{n=1}^{P}\left(1+\alpha_{1} b / n \pi\right) \prod_{n=1}^{Q}\left(1+\alpha_{1} c / n \pi\right)}=G, \text { say }
$$


has the asymptotic behavior

$$
\lim _{\substack{P \rightarrow \infty \\ Q \rightarrow \infty}} G=\frac{1}{\left(1+\alpha_{1} a / r \pi\right)} \cdot \frac{\Gamma\left(1+\alpha_{1} b / \pi\right) \Gamma\left(1+\alpha_{1} c / \pi\right)}{\Gamma\left(1+\alpha_{1} a / \pi\right)}(1+1 / C)^{-\alpha_{1} b / \pi}(1+C)^{-\alpha_{1} c / \pi}
$$

and hence tends to a nonzero constant as $r$ becomes large. We can sum up all this and arrive at the asymptotic behavior

$$
\lim _{\substack{P \rightarrow \infty \\ Q \rightarrow \infty}} A_{r}^{\prime} \simeq 0\left(\frac{1}{r^{3 / 2}} \cdot \frac{(1+1 / C)^{r b / a}(1+C)^{r c / a}}{(1+c / b)^{r b / a}(1+b / c)^{r c / a}}\right) \text { for large } r .
$$

Letting $X=b / c$, we can rewrite (29) after some manipulation as

$$
\lim _{\substack{P \rightarrow \infty \\ Q \rightarrow \infty}} A_{r}^{\prime} \simeq 0\left((r)^{-3 / 2}\left\{\left(\frac{1+C}{1+X}\right) \cdot\left(\frac{X}{C}\right)^{b / a}\right\}^{r}\right) \text { for large } r .
$$

Let $C=P / Q=\sigma X$. Substituting for $C$ and rearranging (30) one may obtain

$$
\left(\frac{1+C}{1+X}\right)\left(\frac{X}{C}\right)^{b / a}=\{1+(\sigma-1) p\} \sigma^{-p}=\eta, \text { say },
$$

with $p=b / a=X /(1+X)$.

Using (31) in (30) we write

$$
\lim _{\substack{P \rightarrow \infty \\ Q \rightarrow \infty}} A_{r}^{\prime} \simeq 0\left((r)^{-3 / 2} \eta^{r}\right)
$$

Without loss of generality, let us let $b>c$ implying $X=b / c \geq 1$ and hence $0.5 \leq p<1$. It is shown in appendix 2 that under this condition

$$
\eta>1 \text { for } \sigma \neq 1
$$

and by direct substitution we see that

$$
\eta=1 \text { for } \sigma=1 \text {. }
$$

Using these in (32), it follows that the higher order coefficients $A_{r}^{\prime}$ have an exponential growth for $\sigma \neq 1$, i.e., for $C=P / Q \neq X=b / c$. Only for $\sigma=1$, which implies $P / Q=b / c, A_{r}^{\prime}$ 's exhibit an algebraic behavior and are $0\left(r^{-3 / 2}\right)$ for large $r$.

\section{The Edge Condition and the Proper Choice of $C$}

The condition at the edge of septum requires that the field potential $\varphi$ go to zero at the edge as $r^{1 / 2}$ and that $|\nabla \varphi|$ go to infinity as $r^{-1 / 2}$. Now it is seen from (2a) that

$$
\left.\frac{\partial \varphi_{A}}{\partial z}\right|_{x=b}=-\alpha_{1} A e^{-\alpha_{1} z} \sin \frac{\pi b}{a}+\sum_{n=1}^{\infty}\left\{A_{n} \sin (n \pi b / a)\right\} \cdot \alpha_{n} e^{\alpha_{n} z}
$$

It is obvious that if $A_{n}^{\prime}=-\sin (n \pi b / a) A_{n} / \sin (\pi b / a)$ increases exponentially for large $n$, which it does when $P / Q=C \neq X=b / c$, the expression for $\partial \varphi_{A} /\left.\partial z\right|_{x=b}$ at the edge goes to infinity in a much stronger manner than $z^{-1 / 2}$ as $z \rightarrow 0$. It is only when $C=X$ and $A_{n}^{\prime}$ is $0\left(1 / n^{3 / 2}\right)$ for large $n$ that the sum of the series is $0\left(z^{-1 / 2}\right)$ as $z \rightarrow 0$, as may be shown by following a method due to Hurd. It may be pointed out that in the exact solution obtained by Hurd $A_{n}^{\prime}$ is $0\left(1 / n^{3 / 2}\right)$ for large $n$, as it of course must be. 
The conclusion is then that the edge condition is satisfied only with the choice of $C=X$, i.e., $P / Q=b / c$. When $P$ and $Q$ are increased indefinitely, consistent with the condition $C=X$, the solution converges to the one which satisfies the required behavior at the edge and thus yields an unique answer satisfying the physical conditions.

\section{Conclusion}

It has been shown that the solution of a doubly infinite set of equations may exhibit relative convergence in that it may be dependent on the manner in which the doubly infinite set is truncated. An additional condition, such as the edge condition, may be used to determine the correct way of truncating the infinite set. Only with the proper choice of the ratio of the number of equations, the solution of the truncated set yields the exact solution as the number of equations is increased indefinitely. Otherwise, the solution converges to an incorrect answer which depends on the ratio of the number of equations from the doubly infinite set.

It is hoped that this work may provide insight for handling a class of problems of a similar type which do not lend themselves to an exact solution.

The author acknowledges the helpful discussions with his colleagues in the Antenna Laboratory of the University of Illinois and the sponsorship of Aeronautical Systems Division, Wright-Patterson Air Force Base, Ohio, through the research grant AF33(616)-6079. He is also thankful to Professor S. A. Schelkunoff for making some helpful comments on the paper. The paper was prepared for publication while the author was a summer employee at the Radar Division, Ground Systems Group, Hughes Aircraft Company, Fullerton, Calif., and he is thankful for all the assistance provided him during the course of the preparation of the paper.

\section{Appendix 1. Convergence of Certain Products}

We shall prove the convergence of the products of the type

$$
\prod_{n=1}^{P} \frac{\left(1-\alpha_{r} a / n \pi\right)}{\left(1-\alpha_{r} / \alpha_{n}\right)}
$$

as $P \rightarrow \infty$. These products appear in (19) in the text. To prove the convergence of the products in $(\mathrm{A}-1)$, it will be sufficient to show that the series $S\left(\alpha_{r}\right)$ given by

$$
S\left(\alpha_{r}\right)=\sum_{n=1}^{\infty}\left\{\ln \left(1-\alpha_{r} a / n \pi\right)-\ln \left(1-\alpha_{r} / \alpha_{n}\right)\right\}
$$

converges. Consider the $R$ th terms of the series for $R$ large such that $\left(\alpha_{r} a / R \pi\right)<<1$. Expand the log functions in a series and obtain

$$
\ln \left(1-\alpha_{r} a / R \pi\right)-\ln \left(1-\alpha_{r} / \alpha_{R}\right)=\alpha_{r}\left(a / R \pi-1 / \alpha_{R}\right)+0\left(1 / R^{2}\right) .
$$

Using $\alpha_{R}=\sqrt{(R \pi / a)^{2}-k^{2}}$ one derives

$$
1 / \alpha_{R}=a / R \pi+0\left(1 / R^{3}\right) .
$$

From $(\mathrm{A}-3)$ and $(\mathrm{A}-4)$, it is obvious that the $R$ th term of the series in $(\mathrm{A}-2)$ is at least $0\left(1 / R^{2}\right)$ and hence the series is convergent. By exponentiating the series, one immediately concludes that the product under consideration is convergent. Similar reasoning applies for the other products appearing in the braces in (19).

To prove the inequality

\section{Appendix 2}

$$
1+(\sigma-1) p>\sigma^{p}, \quad 0.5 \leq p<1
$$


for $\sigma \neq 1$. Note for $\sigma=1$, the above becomes an equality.

We shall take a geometric approach to show that the above is true. The two functions $1+(\sigma-1) p$ and $\sigma^{p}$ are plotted as functions of $\sigma$ in figure 2, and it is seen that they have a common point at $\sigma=1$.

FIGURE 2. Plot of functions appearing in inequality relation.

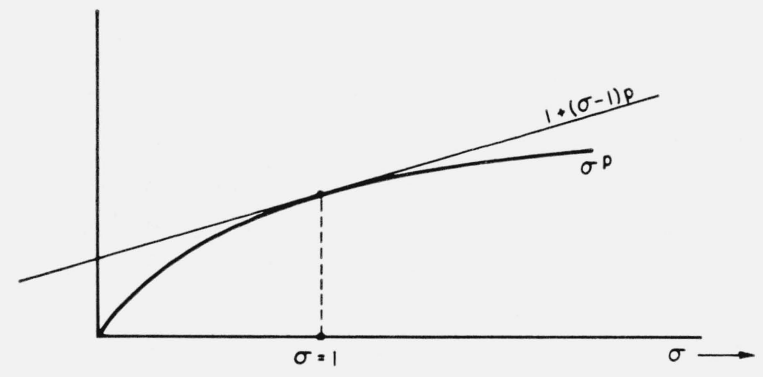

The slope of the curve $\sigma^{p}$ satisfies the following:

Slope of $\sigma^{p}=p \sigma^{p-1}$

$$
\begin{aligned}
& <p \text { for } \sigma>1 \\
& >p \text { for } \sigma<1 \\
& =p \text { for } \sigma=1
\end{aligned}
$$

Since the slope of the straight line is $p$ and the two curves have a common point at $\sigma=1$, it follows from above that the line $1+(\sigma-1) p$ is tangent to the curve $\sigma^{p}$ at $\sigma=1$ and, furthermore, the curve $\sigma^{p}$ lies below this tangent for all $\sigma \neq 1$.

Hence, the inequality is proven.

\section{References}

Bouwkamp, C. J. (Oct. 1946), Singularities at sharp edges in diffraction theory, Physica 12, $467-474$.

Hurd, R. A., and H. Gruenberg (Nov. 1954), H-plane bifurcation of rectangular waveguides, Can. J. Phys. 32, 694-701.

Magnus, W., and F. Oberhetinger (1954), Functions of mathematical physics (Chelsea Publishing Co., New York, N.Y.).

Muir, Thomas (1960). A treatise on the theory of determinants (Dover, New York), 346.

(Paper 67D2-260) 